\title{
Local Structure and Dynamics of Functional Materials Studied by X-ray Absorption Fine Structure
}

\author{
Takafumi Miyanaga
}

Citation: Miyanaga, T. Local

Structure and Dynamics of Functional Materials Studied by X-ray

Absorption Fine Structure. Symmetry

2021, 13, 1315. https://doi.org/

$10.3390 /$ sym 13081315

Academic Editor: Elena Belokoneva

Received: 22 May 2021

Accepted: 5 July 2021

Published: 22 July 2021

Publisher's Note: MDPI stays neutral with regard to jurisdictional claims in published maps and institutional affiliations.

Copyright: (C) 2021 by the author. Licensee MDPI, Basel, Switzerland. This article is an open access article distributed under the terms and conditions of the Creative Commons Attribution (CC BY) license (https:/ / creativecommons.org/licenses/by/ $4.0 /)$.
Department of Mathematics and Physics, Hirosaki University, Hirosaki, Aomori 036-8561, Japan; takaf@hirosaki-u.ac.jp; Tel.: +81-172-39-3551

\begin{abstract}
X-ray absorption fine structure (XAFS) is a powerful technique used to analyze a local electronic structure, local atomic structure, and structural dynamics. In this review, I present examples of XAFS that apply to the local structure and dynamics of functional materials: (1) structure phase transition in perovskite $\mathrm{PbTiO}_{3}$ and magnetic FeRhPd alloys; (2) nano-scaled fluctuations related to their magnetic properties in Ni-Mn alloys and Fe/Cr thin films; and (3) the Debye-Waller factors related to the chemical reactivity for catalysis in polyanions and ligand exchange reaction. This study shows that the local structure and dynamics are related to the characteristic function of the materials.
\end{abstract}

Keywords: XAFS; XMCD; Debye-Waller factor; structure and magnetic phase transition; magnetic alloys; chemical reactivity

\section{Introduction}

Symmetry is one of the most important concepts in natural science. Symmetry lies at the heart of fundamental laws of nature as an important tool for understanding the properties of complex systems, including condensed matter physics, materials science, and technology. The concept of symmetry plays an important role in the phase transition, exotic electronic structure, magnetic structure, and chemical reactivity in condensed matters and chemical systems.

X-ray absorption fine structure (XAFS), which has been developed in recent years accompanying the synchrotron radiation source, is becoming a powerful technique that is used to study the local atomic structure, electronic structure, and structural dynamics of ferroelectric and magnetic materials, semiconductors, molecules, and gas-phase systems. XAFS is a hybrid technique that can be used to study both the structure and electronic state, which is strongly correlated with symmetry in condensed matters. I discuss the physical properties and chemical reactivities of multiple functional materials from the symmetry point of view, which can be a key concept of recent industrial innovation.

The history of XAFS began in the 1970s with the treatise of Lytle, Sayers, Stern [1]. Before the 1920s when the fine structure on X-ray absorption spectra was discovered, many researchers, including Japanese scientists, struggled to interpret its behavior [2,3]. This can be recognized as the starting point of XAFS when considering the physical meaning, and it led to a structural analysis of the materials. Recently, accompanying the development of synchrotron radiation sources and theoretical research, the XAFS technique has seen considerable advances. There are fewer limitations in the measurement setup because the X-ray absorption transmission technique is simple. XAFS is applicable to structure studies of solid, liquid, gas phase, thin films and nanoparticles because the atomic long-range order is not required. XAFS provides local structure information because of the relatively small electron mean free path emitted during the X-ray absorption process. These two characteristic features make XAFS a widely applicable technique, commonly being applied to the following: crystalline and non-crystalline samples, amorphous liquid and solutions, nanoparticles, surfaces, thin films, etc. [4-6]. Additionally, XAFS is an element and electron shell-selective by choosing the appropriate absorption edge. XAFS can be divided into 
two parts: XANES (X-ray absorption near-edge structure) and EXAFS (Extended XAFS). EXAFS includes information about the local structure around the X-ray absorption atom as radial distribution because of the simple theoretical interpretation. On the other hand, the origin of XANES is more complicated than that of EXAFS and has rich information on the electronic structure, valence, and 3D local structure. One of the parameters of the EXAFS signal affected by thermal vibration and structural disorder, the so-called Debye-Waller factor, involves structure dynamics and disorders.

In this paper, I discuss examples of XAFS studies applied to functional materials in which symmetry, or symmetry breaking, plays an important role in the origin of their functions. Section 2 shows a brief introduction of the XAFS method and analysis. In Section 3, structural phase transition in highly symmetric perovskite-type $\mathrm{PbTiO}_{3}$ and $\mathrm{FeRh}(\mathrm{Pd})$ systems with $\mathrm{CsCl}$-type structures are presented. Section 4 shows the application of a circularly polarized X-ray source to a magnetic alloy of Ni-Mn and multilayer of $\mathrm{Fe} / \mathrm{Cr}$. Section 5 shows the application of Debye-Waller factor analysis to chemical reactivities, where the chemical reactivity as a catalyst of Mo polyanion and ligand exchange reaction of $3 d$ metal ion in water are discussed.

\section{Methods and Analysis}

XAFS is an analysis of the fine structure on the absorption edge in X-ray absorption spectra. The basic measurement mode is a transmission mode using, for example, ionization chambers to measure the photon flux before and after the sample. XAFS can also be measured in fluorescence (using semiconducting detector) or electron-yield mode; these techniques are well suited to study thin films, dilute elements, or surface structure. EXAFS formalism is well defined and simple within a single scattering theory [7], but XANES formalism is complicated and uses band calculation and molecular orbital schemes or multiple scattering calculations [8].

From the EXAFS analysis, the interatomic distance from the X-ray absorbing atom to the nearest neighboring atoms as a radial distribution, their coordination numbers, and thermal and structural fluctuation at the distances (so called Debye-Waller factor) can be obtained as the structural information. The XANES region is sensitive to the electronic state of X-ray absorbing and surrounding atoms. XANES is also sensitive to the 3D structure, because the multiple scattering effects are important with long photoelectrons, causing a mean free path at such low-energy photoelectrons.

The Debye-Waller factor in EXAFS includes information from thermal dynamics and structure disorders. Cumulant expansion analysis reveals the anharmonic effect of the thermal vibration and structure disorder $[9,10]$. To obtain the several order cumulants, there are two types of theoretical pictures: the field theoretical method for the small anharmonic effect and the path integral technique, which is applied to a large anharmonic effect, e.g., double-well potential.

\section{Local Structural Change in Phase Transitions in Perovskites and Magnetic Alloys}

In this section, I discuss two types of structural phase transition in $\mathrm{PbTiO}_{3}$ and $\mathrm{FeRh}(\mathrm{Pd})$ magnetic alloys. These two examples have long been studied, and their industrial application is becoming important today. The first example is perovskite-type $\mathrm{PbTiO}_{3}$, in which, among the same type of perovskites, the largest distortion on the tetragonal to cubic transition is known to occur. The second example is the structural change observed in the transition from antiferromagnetic to ferromagnetic in FeRh and FeRhPd alloys, which show a large magnetocaloric effect near room temperature.

\subsection{Structure Phase Transition in Perovskite-Type $\mathrm{PbTiO}_{3}$}

Structural phase transition is an interesting subject in basic physics, and it has recently attracted industrial attention. Perovskite-type materials are important in this phase transition system. In basic physics, these phase transitions are treated as being deeply related to symmetry breaking. The phase transition mechanism in $\mathrm{PbTiO}_{3}$ has been well studied 
by diffraction techniques because of the large distortion of ions along the $c$-axes in the tetragonal phase. Two types of phase transition have been proposed: "displacive type" and "order-disorder type". The displacive type is connected to the actual atomic displacement between two phases. The displacive transition generally involves soft mode, where the frequency of the atomic vibration tends to zero at the critical temperature. However, with the order-disorder type, local atomic displacement is conserved between two phases, but the direction of distortion changes to be randomly oriented. While the diffraction technique based on long-range order (LRO) cannot be easily and clearly distinguished by these two types of transitions. XAFS based on short-range order (SRO) could show local distortions. For example, in the cubic phase, the bond distance of three directions $(x, y, z)$ is the same in the diffraction technique, but XAFS shows different distances in the case of order-disorder transition. From the original neutron and X-ray diffraction study, the so-called displacive transition is proposed in the transition between the tetragonal and cubic phase in $\mathrm{PbTiO}_{3}$, in which the relative displacement of ions disappears at a higher temperature phase. Recently, from high-resolution $\mathrm{X}$-ray diffraction studies, the $\mathrm{Pb}-\mathrm{O}$ bonds show considerably strong covalency in tetragonal $\mathrm{PbTiO}_{3}$, while those bonds in the cubic phase are ionic [11]. However, XAFS is sensitive to local electronic and atomic structures, and it is useful for the detection of such a local atomic displacement. Figure 1a shows the temperature dependence of the area of pre-edge peak (A, narrow peak at $4968 \mathrm{eV}$ ) on Ti K-edge for $\mathrm{PbTiO}_{3}$ [12]. The pre-edge $\mathrm{A}$ is generated from weak quadrupole transition in the $1 s-3 d$ state because dipole transition is forbidden. If the $p$-state from the ligand $\mathrm{O}$ atom hybridizes to the $d$-state of $\mathrm{Ti}$, then a strong pre-edge peak is observed. The mixing of the $p$-state strongly depends on the symmetry around Ti atom; when it is more deviated from $\mathrm{O}_{\mathrm{h}}$ symmetry, the peak becomes larger, so the peak intensity is used as an indication of symmetry. The area of peak A decreases from low temperature to $763 \mathrm{~K}$, but the area value is still largely higher than 0 , even in the cubic phase (higher than $763 \mathrm{~K}$ ).

This phenomenon is also recognized in the interatomic distances obtained from EXAFS analysis. Figure $1 \mathrm{~b}$ shows the temperature dependence of the interatomic distances for three types of $\mathrm{Pb}-\mathrm{O}$ pairs obtained from $\mathrm{Pb}_{\mathrm{III}}$-edge EXAFS [13]. There are completely separated by three different $\mathrm{Pb}-\mathrm{O}$ distances in the tetragonal region (less than $763 \mathrm{~K}$ ). In these three types of atomic pairs, the longest pair of $\mathrm{Pb}-\mathrm{O}(3)$ shows the same behavior as that of the area of peak A shown in Figure 1a. It is noted that there are still three different interatomic distances, even in the cubic phase (higher than $763 \mathrm{~K}$ ). Moreover, the symmetry around the Ti atom is not completely the same as that of the $\mathrm{Pb}$ atom. However the distortion of $\mathrm{O}(3)$ atoms related to the $\mathrm{Pb}$ atom induces the symmetry breaking around the Ti atom. This result shows that there is local distortion, even in the cubic phase as similarly shown in Figure 1a, in which distortion peak A still exists in the cubic phase. The distortion in the cubic phase deduces the order-disorder type transition. As a result, the structural phase transition in $\mathrm{PbTiO}_{3}$ is hybrid type, where the longest $\mathrm{Pb}-\mathrm{O}(3)$ along the $c$-axis is displacive type, and $\mathrm{Pb}-\mathrm{O}(1)$ and $\mathrm{Pb}-\mathrm{O}(2)$ show the order-disorder-type transition. Figure 1c shows the temperature dependence of the Debye-Waller factor for the $\mathrm{Pb}-\mathrm{O}(3)$ atomic pair. The softening behavior, which is the feature of displacive-type transition, can be clearly observed, and this cannot be found in other $\mathrm{Pb}-\mathrm{O}(1,2)$ pairs [12]. The EXAFS revealed characteristic features in the longest $\mathrm{Pb}-\mathrm{O}(3)$ atomic pairs in the structural phase transition of $\mathrm{PbTiO}_{3}$. 

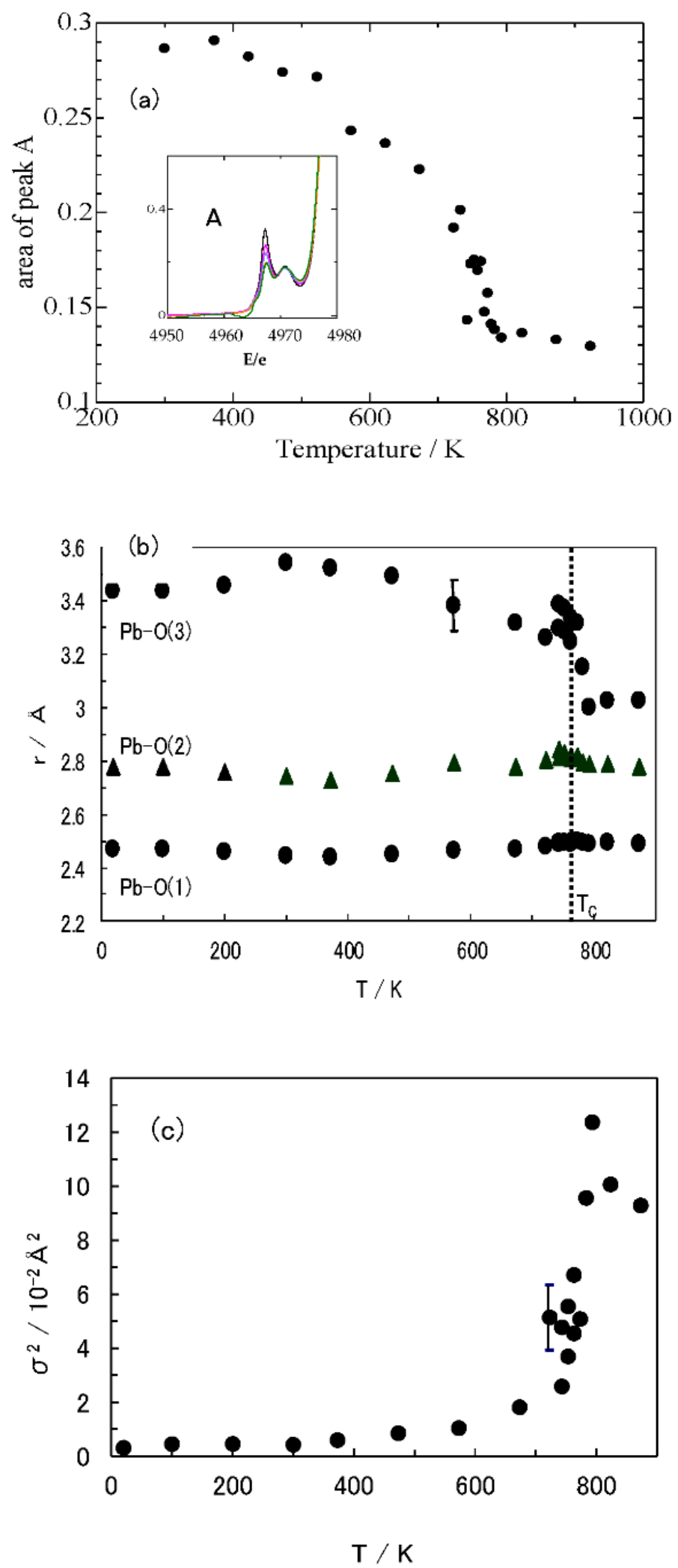

Figure 1. (a) Temperature dependence of the area of the pre-edge peak (narrow peak at $4968 \mathrm{eV}$ ) on Ti K-edge XANES (inset shows the XANES profiles around the pre-edge peak A). (b) Temperature dependences of interatomic distances of three types of $\mathrm{Pb}-\mathrm{O}(1,2,3)$ in $\mathrm{PbTiO}_{3}$ obtained from the $\mathrm{Pb}$ $\mathrm{L}_{\mathrm{III}}$-edge EXAFS. (c) Temperature dependence of the Debye-Waller $\left(\sigma^{2}\right)$ factor of $\mathrm{Pb}-\mathrm{O}(3)$ atomic pair

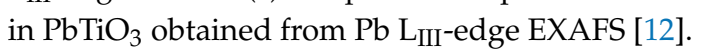




\subsection{Structure Phase Transition in FeRh and FeRhPd Magnetic Alloys}

FeRh magnetic alloys exist as a chemically ordered phase near the equiatomic composition, and the ordered alloy shows a first-order phase transition from an antiferromagnetic (AFM) to a ferromagnetic (FM) phase around $T_{p}=370 \mathrm{~K}$ [14]. This system has also long been studied by those with an interest in basic physics. However, it is still a hot topic because of the application to the magnetic refrigerator [15]. Accompanying the magnetic transition in this temperature region is a sharp thermal expansion that can be observed, but there is no satisfied model in which either magnetic transition affects the structural one or vice versa. It is still an open question similar to the "egg or chicken" problem. Figure 2a,b show the temperature dependence of the Fourier transform (FT) for Fe K-edge and Rh K-edge EXAFS for $\mathrm{Fe}_{50.4} \mathrm{Rh}_{49.6}$, respectively. After the detailed fitting analysis, the atomic distances can be obtained, and their temperature dependences are shown in Figure 2c. I assume that the bcc structure is preserved over the transition, and $\mathrm{Fe}-\mathrm{Rh}$ (or $\mathrm{Rh}-\mathrm{Fe}$ ) is plotted by times $2 / \sqrt{3}$ to compare $\mathrm{Fe}-\mathrm{Fe}$ (or $\mathrm{Rh}-\mathrm{Rh}$ ). The behavior of $\mathrm{Fe}-\mathrm{Fe}$ is almost the same as that of Rh-Rh. It is strange that the lattice distance of $\mathrm{Fe}-\mathrm{Rh}$ (or $\mathrm{Rh}-\mathrm{Fe}$ ) is shorter than that of $\mathrm{Fe}-\mathrm{Fe}$ (or Rh-Rh) [14]. A similar discrepancy has been observed in the XAFS study of FeRh thin films [16]. The behavior of Fe-Fe (or Rh-Rh) and Fe-Rh (Rh-Fe) is different. While the sharp expansion for Fe-Rh (or Rh-Fe) starts at $250 \mathrm{~K}\left(\mathrm{~T}_{1}\right)$ and finishes at $350 \mathrm{~K}\left(\mathrm{~T}_{2}\right)$, that for $\mathrm{Fe}-\mathrm{Fe}$ (or Rh-Rh) finishes at $400 \mathrm{~K}\left(\mathrm{~T}_{3}\right)$. To date, the mechanism of this interesting phenomenon remains unsolved, but one interpretation was proposed that the local distortion to (111) direction starts at $\mathrm{T}_{1}$ and finishes at $\mathrm{T}_{2}$; in the distorted phase, $\mathrm{Fe}-\mathrm{Rh}$ (or $\mathrm{Rh}-\mathrm{Fe}$ ) distance is shorter than $\mathrm{Fe}-\mathrm{Fe}$ (or $\mathrm{Rh}-\mathrm{Rh}$ ) distance because of the EXAFS two-shell fit. At the temperature range between $\mathrm{T}_{2}$ and $\mathrm{T}_{3}$, the unchanged distortion shows only thermal expansion [14].
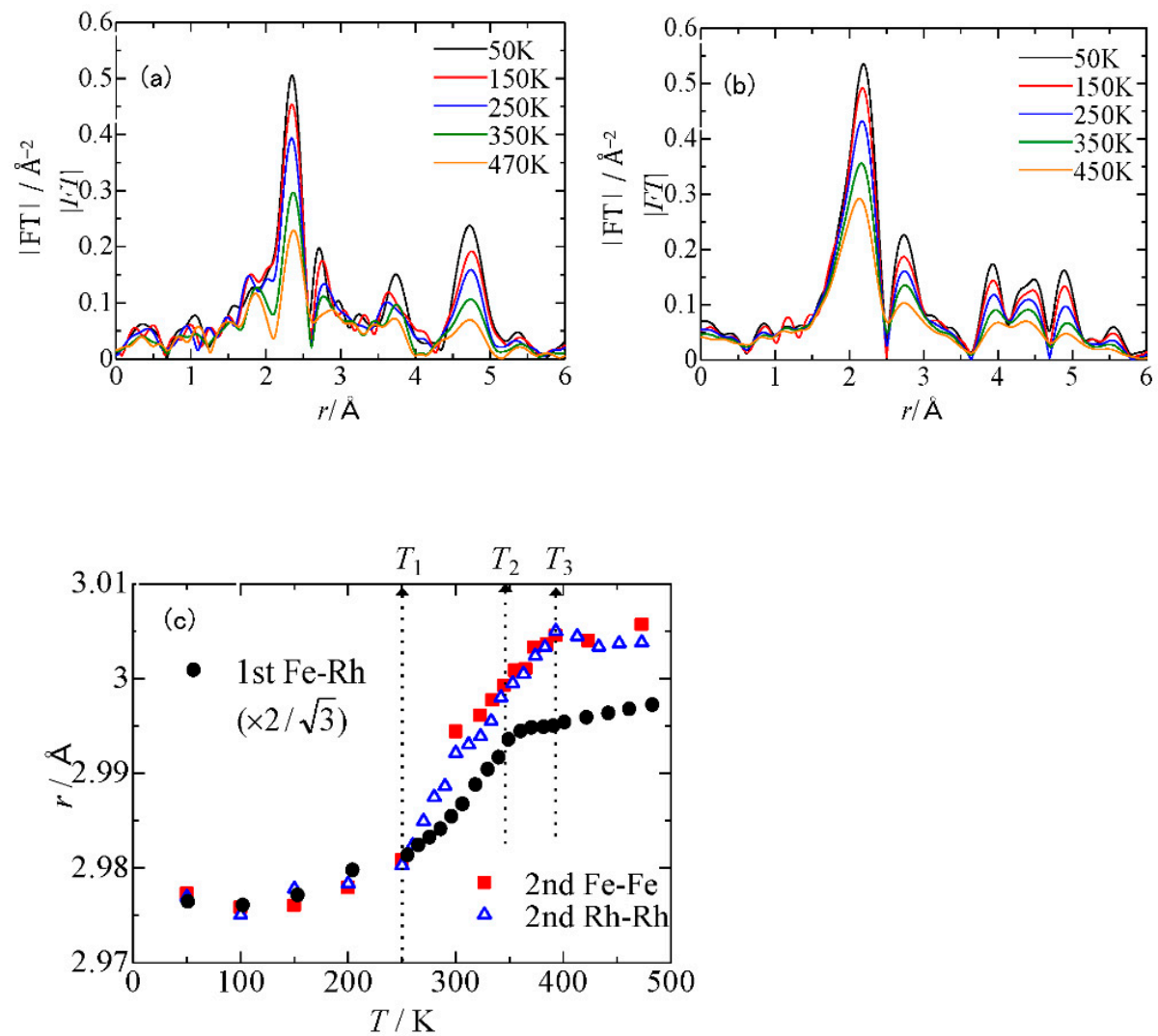

Figure 2. EXAFS Fourier transforms of (a) Fe K- and (b) Rh K-edge EXAFS, and (c) temperature dependences of interatomic distances obtained from EXAFS curve fitting for ordered $\mathrm{Fe}_{50.4} \mathrm{Rh}_{49.6}$ alloy. The interatomic distance of Fe-Rh (or Rh-Fe) is plotted by times $2 / \sqrt{3}$ to compare it with that of Fe-Fe (or Rh-Rh) [14]. 
When a small portion of $\mathrm{Rh}$ atoms are replaced by $\mathrm{Pd}$ atoms, the magnetization increases and $\mathrm{T}_{\mathrm{p}}$ shifts to a lower temperature. Fe and Pd K-edge EXAFS for $\mathrm{Fe}_{49.7} \mathrm{Rh}_{47.4} \mathrm{Pd}_{2.9}$ and $\mathrm{Fe}_{48.3} \mathrm{Rh}_{46.8} \mathrm{Pd}_{4.9}$ alloys were discussed as follows: (1) local distortion as found in $\mathrm{FeRh}$, and (2) the role of Pd atoms in changing the magnetic properties [17]. Figure $3 \mathrm{a}, \mathrm{b}$ show $\mathrm{k} \chi(\mathrm{k})$ and the Fourier transform of Pd K-edge EXAFS for $\mathrm{Fe}_{49.7} \mathrm{Rh}_{47.4} \mathrm{Pd}_{2.9}$ alloys for several temperatures. The original FeRh alloy has a bcc- $\mathrm{CsCl}$ structure, and $\mathrm{Rh}$ atoms are considered to be replaced by $\mathrm{Pd}$ atoms in $\mathrm{Fe}_{49.7} \mathrm{Rh}_{47.4} \mathrm{Pd}_{2.9}$ alloys. The interatomic distances of $\mathrm{Fe}-\mathrm{Rh}$ and $\mathrm{Fe}-\mathrm{Fe}$ obtained from the Fe K-edge XAFS analysis for $\mathrm{Fe}_{49.7} \mathrm{Rh}_{47.4} \mathrm{Pd}_{2.9}$ and $\mathrm{Fe}_{48.3} \mathrm{Rh}_{46.8} \mathrm{Pd}_{4.9}$ are about $0.02 \AA$ longer than that from $\mathrm{Fe}_{50.4} \mathrm{Rh}_{49.6}$ [17]. These results can be estimated from the lattice constant of $\mathrm{XRD}$, which means that the local structure around Fe atoms from XAFS is almost the same as the average structure from XRD. However, the interatomic distances of $\mathrm{Pd}-\mathrm{Fe}$ and $\mathrm{Pd}-\mathrm{Rh}$ obtained from the Pd K-edge XAFS analysis for $\mathrm{Fe}_{49.7} \mathrm{Rh}_{47.4} \mathrm{Pd}_{2.9}$ and $\mathrm{Fe}_{48.3} \mathrm{Rh}_{46.8} \mathrm{Pd}_{4.9}$ are about $0.04 \AA$ longer than that from $\mathrm{Fe}_{50.4} \mathrm{Rh}_{49.6}$ [17]. This result means that the local structure around the Pd atom is more expanded than that around $\mathrm{Rh}$ atoms in FeRhPd alloys. The expansion around Pd atoms is expected to play an important role in the characteristic magnetic properties in $\mathrm{FeRhPd}$, e.g., decreasing transition temperature, $\mathrm{T}_{\mathrm{p}}$, as $\mathrm{Pd}$ doping.
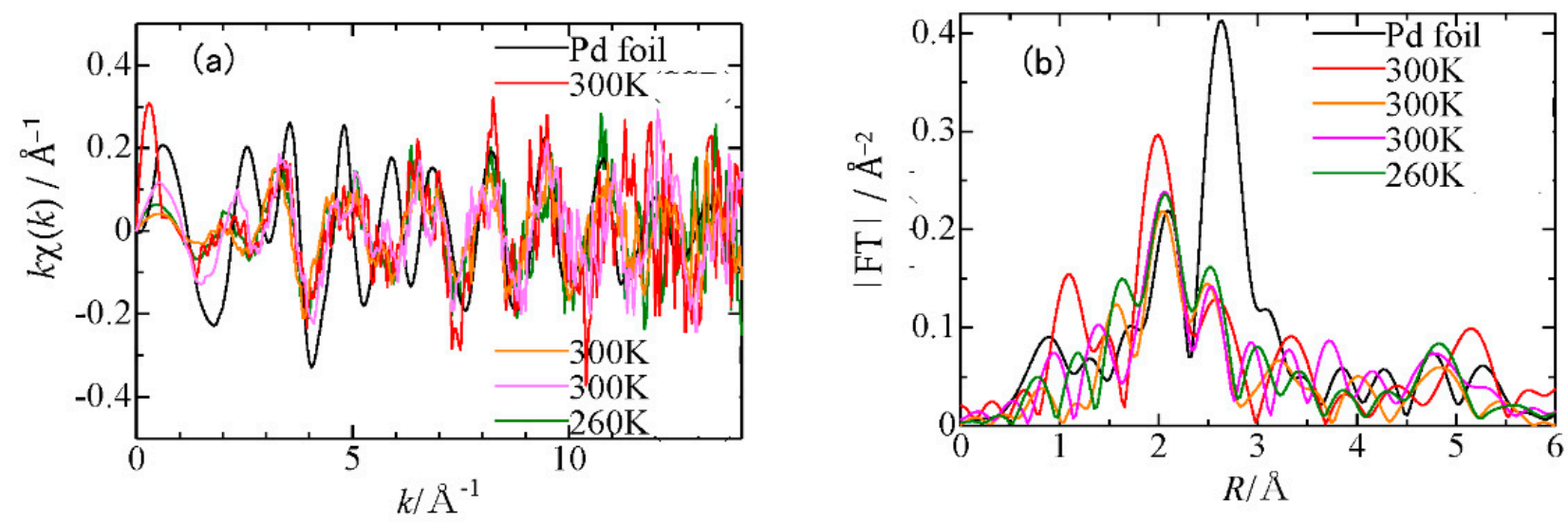

Figure 3. (a) $k \chi(k)$ and (b) Fourier transforms of Pd K-edge EXAFS for $\mathrm{Fe}_{49.7} \mathrm{Rh}_{47.4} \mathrm{Pd}_{2.9}$ alloy for several temperatures. The result of Pd metal (black line) is also included.

\section{Study of Local Structures of Magnetic Alloys and Thin Films}

In this section, I discuss the application of magnetic EXAFS and X-ray magnetic circular dichroism (XMCD) to magnetic alloys of Ni-Mn and a multilayer of $\mathrm{Fe} / \mathrm{Cr}$, in which atomic ordering is important to characteristic magnetic features.

\subsection{Magnetic EXAFS for $\mathrm{Ni}_{3} \mathrm{Mn}$ Alloys}

$\mathrm{Ni}_{3} \mathrm{Mn}$ magnetic alloys have also been a long-studied system, where they have a coupling between the structure disorder and magnetic properties. I studied the magnetic EXAFS for Mn using circular polarized X-ray, and the following is the discussion of the effect of atomic arrangement in order-disorder couplings to magnetic properties [18,19]. In the magnetic EXAFS, the amplitude is proportional not only to the coordination number but also to the magnetic moment of X-ray absorbing and photoelectron scattering atoms because of spin-orbit coupling. Figure 4 is the Fourier transforms for Mn K-edge of (a) conventional EXAFS and (b) magnetic EXAFS for $\mathrm{Ni}_{0.75} \mathrm{Mn}_{0.25}$ alloy, which was heat-treated at $693 \mathrm{~K}$ in 500 hours to order the atomic arrangement $(S=0.78)$ [18]. In the ordered $\mathrm{Ni}_{3} \mathrm{Mn}$ alloy, the Mn atom has a large magnetic moment (about $3.15 \mu_{\mathrm{B}}$ [18]) and is located at the second nearest neighbor (NN) and fourth $\mathrm{NN}$ from the X-ray absorbing $\mathrm{Mn}$ atom. The FT modulus peak intensity of the second and fourth NN in Figure $4 \mathrm{~b}$ becomes larger than that in Figure 4a because of the large magnetic moment of Mn. From the ratio of the intensities of the first $(\mathrm{Ni})$ and second $(\mathrm{Mn})$ peaks, the ratio of the magnetic moment 
can be evaluated. Table 1 shows the result of magnetization, LRO of the sample, and the ratio of the magnetic moment $(\mathrm{Mn} / \mathrm{Ni})$ for several annealing conditions. From our band calculation, the theoretical value of the magnetic moment ratio, $\mathrm{Mn} / \mathrm{Ni}$, is 5.2 , which is close to the magnetic EXAFS result for the $500 \mathrm{~h}$ annealing sample $(\mathrm{Mn} / \mathrm{Ni}=5.9)$ [19]. The experimental result shows that the $\mathrm{Mn} / \mathrm{Ni}$ magnetic moment increases for a longer annealing time, or further ordering proceeds. In another study, from giant magnetoresistance (GMR) experiments [20], the cluster size of the ordered ferromagnetic $\mathrm{Ni}_{3} \mathrm{Mn}$ phase becomes larger for a longer annealing time, or further ordering proceeds. These results indicate that the magnetic moment of $\mathrm{Mn}$ atoms in the highly ordered $\mathrm{Ni}_{3} \mathrm{Mn}$ phase is larger than that in the less ordered phase.
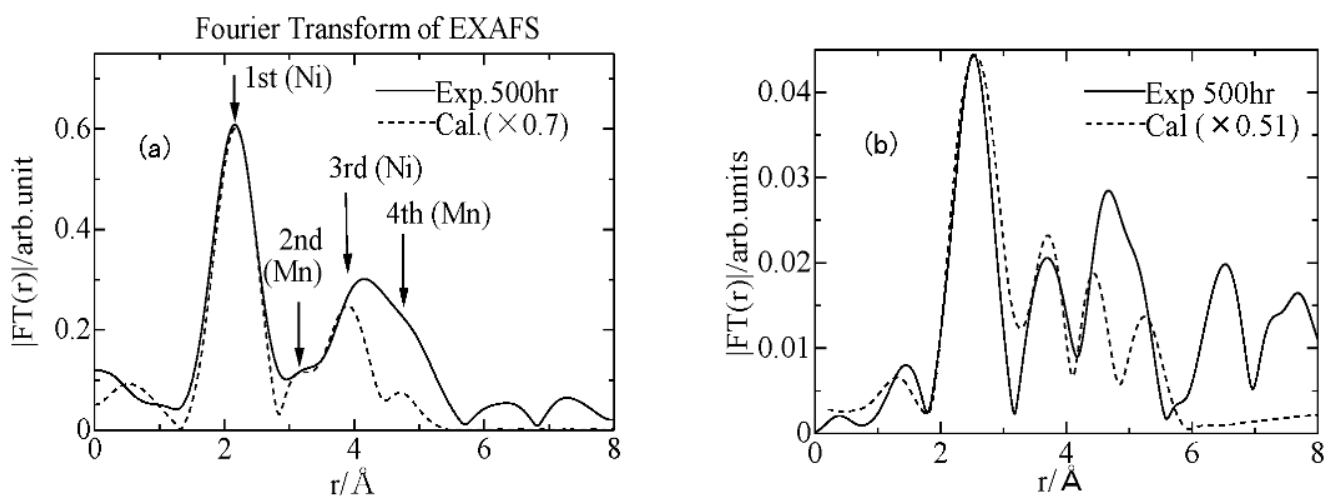

Figure 4. Mn K-edge Fourier transforms of (a) non-magnetic and (b) magnetic EXAFS for ordered (500 h annealing) $\mathrm{Ni}_{0.75} \mathrm{Mn}_{0.25}$ alloy. Dotted lines are the theoretical calculation spectra.

Table 1. Sample magnetization, long-range order (LRO), and the ratio of the atomic magnetic moment of $\mathrm{Mn} / \mathrm{Ni}$ for the samples with several annealing times.

\begin{tabular}{cccc}
\hline Anneal (h) & Magnetization $(\mathbf{e m u} / \mathbf{g})$ & LRO & Ratio Mn/Ni \\
\hline 50 & 50.0 & 0.44 & 3.5 \\
100 & 56.4 & 0.55 & 4.3 \\
500 & 85.9 & 0.78 & 5.9 \\
\hline
\end{tabular}

\subsection{XAFS and XMCD Study for Cluster-Layered Fe/Cr Films}

For the Fe/Cr multilayered structure, GMR was discovered in 1988 [21,22]. In such multilayer structures and granular thin films with ferromagnetic nanoparticles, the correlation between magnetic and transport properties is actively studied [23-25]. In 2006, a minimum point $(\sim 170 \mathrm{~K})$ in the temperature dependence of the resistivity (see Figure $5 \mathrm{a}$ ) was observed in cluster-layered Fe/Cr nanostructures [26]. In this ultrathin film, the small portion of Fe exists as a ferromagnetic cluster in $\mathrm{Cr}$ and indicates superparamagnetic features but no GMR behavior. Local magnetic and atomic structures of cluster-layered $\mathrm{Fe} / \mathrm{Cr}$ thin films were studied by Fe LII, III-edge XMCD and Fe K-edge EXAFS, respectively [27]. Figure $5 \mathrm{~b}$ shows the temperature dependence of the total magnetic moment obtained from the sum rule for Fe $\mathrm{L}_{\mathrm{II}}$, III-edge XMCD of $\mathrm{Al}_{2} \mathrm{O}_{3} / \mathrm{Cr}(70 \AA) /[\mathrm{Fe}(1.2 \AA) / \mathrm{Cr}(10.5 \AA)]_{30} / \mathrm{Cr}(12 \AA)$. The direction between the normal direction of the sample film surface and $X$-ray beam direction, $\theta$, is set to 55 degrees. The magnetic moment of Fe atoms decreases as the temperature increases and disappears above the temperature of minimum resistivity.

From the Fe K-edge EXAFS, it appears that the local structure of this film around Fe atoms for $\mathrm{Al}_{2} \mathrm{O}_{3} / \mathrm{Cr}(70 \AA) /[\mathrm{Fe}(1.2 \AA) / \mathrm{Cr}(10.5 \AA)]_{30} / \mathrm{Cr}(12 \AA)$ is rather disordered, which can be related to the magnetic fluctuations estimated from the theoretical treatment of the temperature dependence of magnetic moments [27]. 

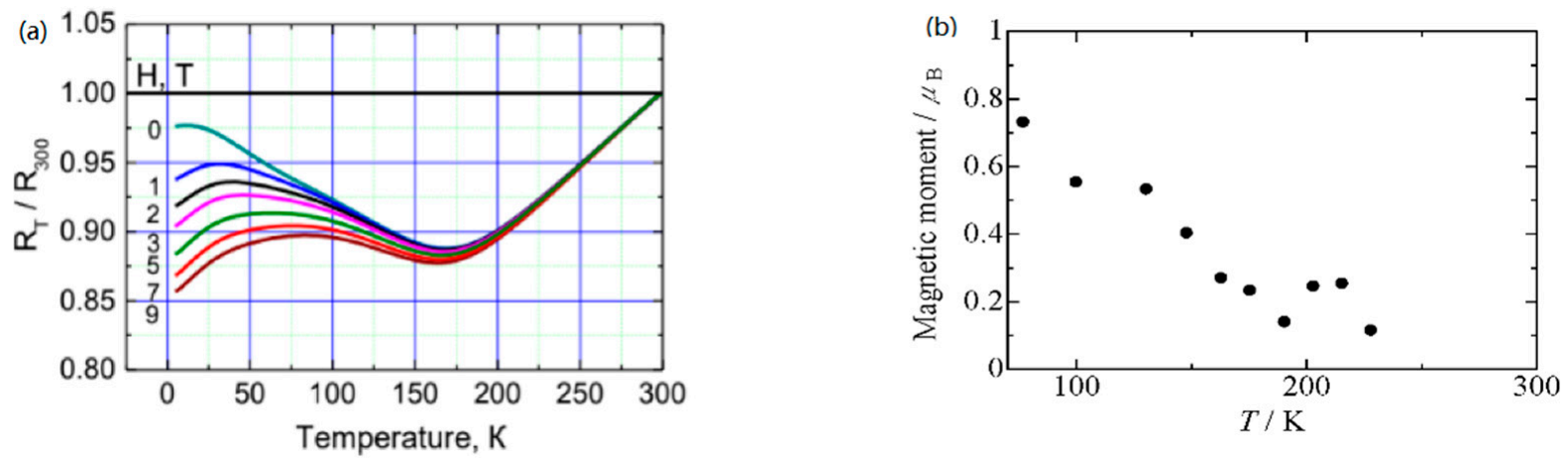

Figure 5. Temperature dependence of relative resistivity for several magnetic fields (a) and that of the total magnetic moment of Fe atoms obtained from XMCD at $\theta=55$ degrees (b).

\section{Structure Dynamics in Chemical Reaction Systems}

In this section, I discuss the structural disorder and dynamics in chemical reaction systems via an analysis of the Debye-Waller factor. The first example is Mo hexamer polyanion. The large fluctuation of the bond distance of the bridging Mo-O-Mo bond is related to chemical reactivity as catalysis. The second example is the ligand exchange reaction, in which an anharmonic potential (or dissociation energy) of $3 d$ metal ion and ligand $\mathrm{H}_{2} \mathrm{O}$ is obtained from the cumulant analysis of EXAFS. This is related to the ligand exchange reaction rate.

\subsection{Structural Disorder in Polyanion of Mo and W}

Mo and $\mathrm{W}$ produce the same type of polyanions, such as $\mathrm{M}_{6} \mathrm{O}_{19}{ }^{2-}$ (see Figure 6a), and show interesting catalytic properties. These Mo and $\mathrm{W}$ polyanion hexamers are highly symmetric structures, and their EXAFS shows interesting behavior $[28,29]$. Figure $6 \mathrm{~b}$ shows the EXAFS Fourier transform for $\mathrm{M}_{6} \mathrm{O}_{19}{ }^{2-}(\mathrm{M}=\mathrm{Mo}$ and $\mathrm{W})$. It is interesting that while the FT peak intensities of Mo-Mo and $\mathrm{W}-\mathrm{W}$ are similar, as expected from the structure data, those of $\mathrm{Mo}-\mathrm{O}$ and $\mathrm{W}-\mathrm{O}$ are different- $\mathrm{W}-\mathrm{O}$ seems to be normal, but $\mathrm{Mo}-\mathrm{O}$ is unnaturally small, although they have a similar symmetric structure. To investigate the reason, I measured the temperature dependence of the Debye-Waller factor and analyzed individual atomic pairs. Figure $6 c, d$ show temperature dependence of the Debye-Waller factor for $\mathrm{Mo}_{6} \mathrm{O}_{19}{ }^{2-}$ and $\mathrm{W}_{6} \mathrm{O}_{19}{ }^{2-}$, respectively. While there are three types of $\mathrm{M}-\mathrm{O}$ bonds in the hexamer, only the Debye-Waller factor of the bridging Mo-O2 is abruptly large. More interestingly, the temperature inclination of $\mathrm{Mo}-\mathrm{O} 2$ is the same as that of other atomic pairs, but the absolute value of this bond is large. This means that the structural disorder of the $\mathrm{Mo}-\mathrm{O} 2$ bond is abnormally large in comparison to that of other atomic pairs. The Jahn-Teller effect of the $\mathrm{Mo}-\mathrm{O} 2$ bond has been reported and suggests an alternative $\mathrm{Mo}-\mathrm{O}=\mathrm{Mo}$ bond distance in $\mathrm{Mo}_{6} \mathrm{O}_{19}{ }^{2-}$ [30]. This larger disorder in bridging the $\mathrm{Mo}-\mathrm{O} 2$ bond may come from the Jahn-Teller effect and should affect the high chemical reactivity of this species.

\subsection{Ligand Exchange Reaction in 3d Transition Metal Ions in Water}

Finally, I introduce the EXAFS application to ligand exchange reactions [31,32]. It is known that $3 d$ transition metal ions exist as hydrated $\mathrm{M}\left(\mathrm{H}_{2} \mathrm{O}\right)^{\mathrm{n}+}$ ions in the water solutions, and ligand $\mathrm{H}_{2} \mathrm{O}$ molecules continuously exchange with water $\mathrm{H}_{2} \mathrm{O}$. These exchange rates are widely distributed from $10^{-6} \mathrm{~s}$ to $10^{8} \mathrm{~s}$. I obtained a harmonic potential of $\mathrm{M}-\left(\mathrm{OH}_{2}\right)$ from the temperature dependence of the Debye-Waller factor in EXAFS for M-O pairs using the relation between the anharmonic potential and higher order cumulant of the thermal factor. As the anharmonic potential of $\mathrm{M}-\left(\mathrm{OH}_{2}\right)$, simple Morse potential was assumed, such as

$$
V(x)=D\left(e^{-2 \alpha x}-2 e^{-\alpha x}\right)
$$


(a)

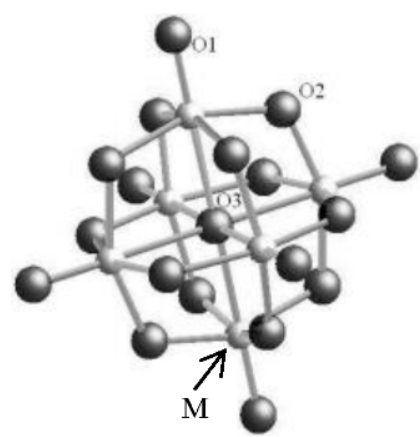

(c)

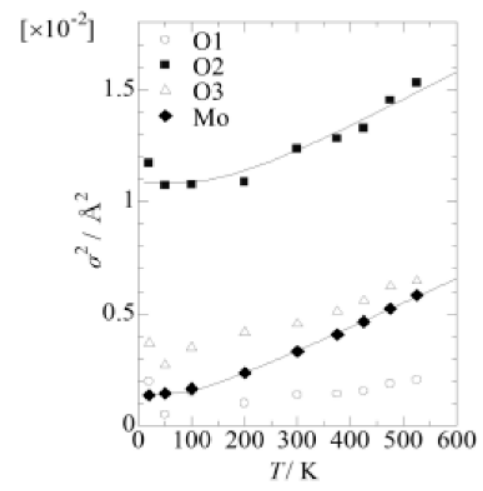

(b)

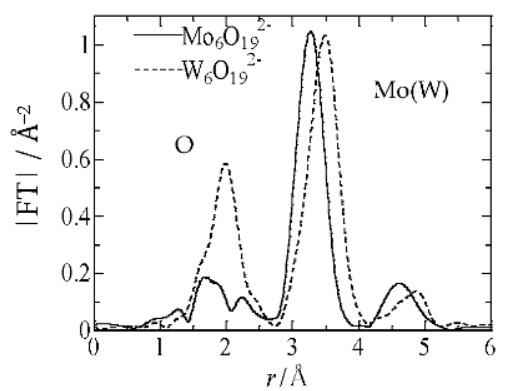

(d)

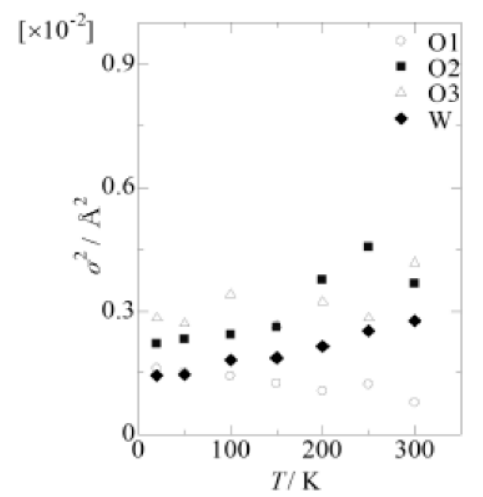

Figure 6. (a) Molecular structure of $\mathrm{M}_{6} \mathrm{O}_{19}{ }^{2-}(\mathrm{M}=\mathrm{Mo}, \mathrm{W})$ compound, (b) EXAFS Fourier transforms of Mo K- and $\mathrm{W} \mathrm{L}_{\mathrm{III}}$-edge EXAFS for $\mathrm{M}_{6} \mathrm{O}_{19}{ }^{2-}(\mathrm{M}=\mathrm{Mo}$ for a solid line, $\mathrm{W}$ for dashed line), (c) temperature dependence of the Debye-Waller factors for $\mathrm{M}-\mathrm{O}$ and $\mathrm{M}-\mathrm{M}$ for $\mathrm{Mo}_{6} \mathrm{O}_{19}{ }^{2-}$, and (d) that for the $\mathrm{W}_{6} \mathrm{O}_{19}{ }^{2-}$ compound (O1: terminal oxygen, $\mathrm{O} 2$ : bridged oxygen, $\mathrm{O} 3$ : center oxygen). Reprinted with permission from [29].

From the temperature dependence of the second- and third-order cumulants, dissociation energy, $D$, and the anharmonic parameter, $\alpha$, can be obtained [32]. Figure 7 shows the plot of $D$ values as a function of the logarithm of the ligand exchange reaction rate. There is a clear correlation between $D$ and the exchange rate. In Figure $7, \times$ represents the result obtained from molecular orbital calculation [33]. The experimental result from EXAFS is slightly smaller than the theoretical one, but their inclinations are similar. It was shown that the ligand exchange rate is strongly related to the anharmonic metal-ligand potential.

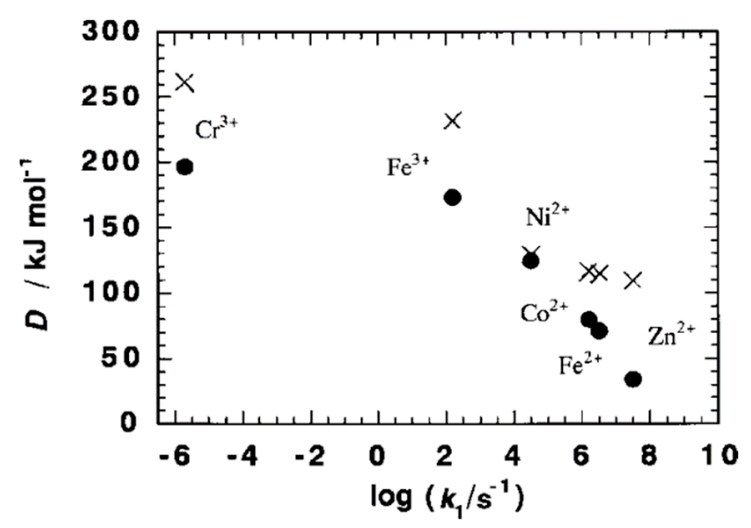

Figure 7. Plots of the dissociation energy, $D$, of a water molecule obtained from EXAFS anharmonic analysis as a function of the water exchange reaction rate $(\bullet)$. The result from the molecular orbital calculation is also presented $(\times)$ Reprinted with permission from [32]. 


\section{Conclusions}

In this review, XAFS applications to local structures and dynamics of functional materials were presented. The materials discussed here are classified into three types of subjects: (1) local structure change in phase transitions of perovskites and magnetic alloys, (2) study of local structures of magnetic alloys and thin films, and (3) structure dynamics in chemical reaction systems. These physical and chemical properties are strongly related to atomic displacement or arrangement.

In structure phase transition, XAFS is sensitive to specific evolutions, such as softmode evolutions or distortion in a specific direction. Magnetic EXAFS and XMCD studies using circular polarized X-ray are sensitive to the magnetic moment of individual atoms in the alloys and thin films. The Debye-Waller factor in EXAFS, which includes the effect of structural distortion and atomic vibration, is connected to the chemical reactivity.

It is shown that XAFS is a local probe and that it is sensitive to a local change in atomic and magnetic structure and structural dynamics, which affect the functions of the materials. Although XAFS has the advantage of allowing for the study of local structure, physical and chemical properties are related to not only SRO but also LRO. In the future, XAFS will continue to be an attractive research technique complementary to the diffraction techniques in material sciences.

Funding: This work was funded by JPSJ KAKENHI under grant numbers JP16K05011 and JP20K05295.

Institutional Review Board Statement: Not applicable.

Informed Consent Statement: Not applicable.

Data Availability Statement: Not applicable.

Acknowledgments: I acknowledge helpful discussions with T. Fujikawa, T. Okazaki, I. Watanabe, D. Diop, Y.A. Babanov, and V.I. Zverev.

Conflicts of Interest: The authors declare no conflict of interest.

\section{References}

1. Sayers, D.; Stern, E.A.; Lytle, F. New Technique for Investigating Noncrystalline Structures: Fourier Analysis of the Extended X-ray-Absorption Fine Structure. Phys. Rev. Lett. 1971, 27, 1204-1207. [CrossRef]

2. Lytle, F.W. The EXAFS family tree: A personal history of the development of extended X-ray absorption fine structure. $J$. Synchrotron Radiat. 1999, 6, 123-134. Available online: http:/ / www.exafsco.com/techpapers / (accessed on 8 July 2021). [CrossRef] [PubMed]

3. Sawada, M.; Tsutsumi, K.; Shiraiwa, T.; Obashi, M. On the Fine Structures of X-ray Absorption Spectra of Amorphous Substances The Amorphous State of the Binary System of Nickel-Sulfur. II. J. Phys. Soc. Jpn. 1955, 10, 464-468. [CrossRef]

4. Schnohr, C.S.; Ridgway, M.C. (Eds.) X-ray Absorption Spectroscopy of Semiconductors; Springer: Berlin/Heidelberg, Germany, 2015.

5. Bunker, G. Introduction to XAFS; Cambridge University Press: Cambridge, UK, 2010.

6. Fornasini, P. Synchrotron Radiation: Basics, Methods and Applications; Mobilio, S., Boscherini, F., Meneghini, C., Eds.; Springer: Berlin/Heidelberg, Germany, 2015; pp. 181-211.

7. Stöhr, J. NEXAFS Spectroscopy; Springer: Berlin/Heidelberg, Germany, 1992.

8. Hatada, K.; Hayakawa, K.; Benfatto, M.; Natoli, C.R. Full-potential multiple scattering for x-ray spectroscopies. Phys. Rev. B 2007, 76, 060102. [CrossRef]

9. Bunker, G. Application of the ratio method of EXAFS analysis to disordered systems. Nucl. Instrum. Methods Phys. Res. 1983, 207, 437-444. [CrossRef]

10. Miyanaga, T.; Fujikawa, T. XAFS Spectroscopy: Basic theory and recent developments. J. Spectrosc. Dyn. $2011,1,4$.

11. Kuroiwa, Y.; Aoyagi, S.; Sawada, A.; Harada, J. Evidence for Pb-O covalency in tetragonal $\mathrm{PbTiO}_{3}$. Phys. Rev. Lett. 2001, 87, 217601. [CrossRef] [PubMed]

12. Miyanaga, T.; Sato, K.; Ikeda, S.; Diop, D. Structural Phase Transition in $\mathrm{PbTiO}_{3}$ from Local Point of View. Recent Res. Dev. Phys. Transw. Res. Netw. Part II 2002, 3, 641-657.

13. Miyanaga, T.; Diop, D.; Ikeda, S.; Kon, H. Study of the Local Structure Changes in $\mathrm{PbTiO}_{3}$ by Pb L $\mathrm{L}_{\mathrm{III}}$ EXAFS. Ferroelectrics 2002, 274, 41-53. [CrossRef]

14. Miyanaga, T.; Itoga, T.; Okazaki, T.; Nitta, K. Local structural change under antiferro-and ferromagnetic transition in FeRh alloy. J. Phys. Conf. Ser. 2009, 190, 012097. [CrossRef] 
15. Zverev, V.I.; Saletsky, A.M.; Gimaev, R.R.; Tishin, A.M.; Miyanaga, T.; Stanton, J.B. Influence of structural defects on the magnetocaloric effect in the vicinity of the first order magnetic transition in $\mathrm{Fe}_{50.4} \mathrm{Rh}_{49.6}$. Appl. Phys. Lett. 2016, $108,192405$. [CrossRef]

16. Wakisaka, Y.; Uemura, Y.; Yokoyama, T.; Asakura, H.; Morimoto, H.; Tabuchi, M.; Ohshima, D.; Kato, T.; Iwata, S. Anomalous structural behavior in the metamagnetic transition of FeRh thin films from a local viewpoint. Phys. Rev. B 2015, $92,184408$. [CrossRef]

17. Komlev, A.S.; Karpenkov, D.Y.; Gimaev, R.R.; Chirkova, A.; Akiyama, A.; Miyanaga, T.; Hupalo, M.F.; Aguiar, D.J.M.; Carvalho, A.M.G.; Jiménez, M.J.; et al. Coupling of magnetic and structural properties in palladium-doped FeRh alloys. to be submitted.

18. Miyanaga, T.; Okazaki, T.; Maruko, R.; Takegahara, K.; Nagamatsu, S.; Fujikawa, T.; Kon, H.; Sakisaka, Y. Magnetic X-ray Absorption Fine Structure for Ni-Mn Alloys. J. Synchrotron Rad. 2003, 10, 113-119. [CrossRef]

19. Miyanaga, T.; Ogasawara, T.; Okazaki, T.; Sakisaka, Y.; Okamoto, K.; Nagamatsu, S.; Fujikawa, T. Local magnetic structures of Ni-Mn alloys in ordered and disordered states studied by magnetic XAFS. J. Magn. Magn. Mater. 2007, 310, e601-e603. [CrossRef]

20. Okazaki, T.; Miyanaga, T.; Sakisaka, Y.; Sugimoto, S.; Yamada, K.; Honda, Z. GMR and soft magnetic properties of Ni-Mn alloys with dispersed ferromagnetic nano particles. Rev. Adv. Mater. Sci. 2004, 6, 150-161.

21. Baibich, M.N.; Broto, J.M.; Fert, A.; Nguyen Van Dau, F.; Petroff, F.; Eitenne, P.; Creuzet, G.; Friederich, A.; Chazelas, J. Giant Magnetoresistance of (001)Fe/(001)Cr Magnetic Superlattices. Phys. Rev. Lett. 1988, 61, 2472-2475. [CrossRef]

22. Parkin, S.S.P.; More, N.; Roche, K.P. Exchange-biased magnetic tunnel junctions and application to nonvolatile magnetic random access memory. Phys. Rev. Lett. 1990, 64, 5852. [CrossRef]

23. Sefrioui, Z.; Mendez, J.L.; Navarro, E.; Cebollada, A.; Briones, F.; Crespo, P.; Hernando, A. Correlation between magnetic and transport properties in nanocrystalline Fe thin films: A grain-boundary magnetic disorder effect. Phys. Rev. B 2001, 64, 224431. [CrossRef]

24. Majumdar, A.K.; Hebard, A.F.; Singh, A.; Temple, D. Extraordinary Hall effect in Fe-Cr giant magnetoresistive multilayers. Phys. Rev. B 2003, 68, 144405. [CrossRef]

25. Almeida, B.G.; Sousa, J.B.; Schad, R.; Moshchalkov, V.V.; Bruynseraede, Y. Phonon-assisted sd electron scattering in Fe/Cr multilayers. J. Magn. Magn. Mater. 1996, 157/158, 730-732. [CrossRef]

26. Ustinov, V.V.; Romashev, L.N.; Milayev, M.A.; Korolev, A.V.; Krinitsina, T.P.; Burkhanov, A.M. Kondo-like effect in the resistivity of superparamagnetic cluster-layered Fe/Cr nanostructures. J. Magn. Magn. Mater. 2006, 300, 148-152. [CrossRef]

27. Miyanaga, T.; Ikeda, Y.; Hasunuma, Y.; Ponomarev, D.; Grebennikov, V.; Babanov, Y.A. Local Magnetic Study for Cluster-Layered Fe/Cr Nanostructures. J. Phys. Conf. Ser. 2019, 1389, 012146. [CrossRef]

28. Miyanaga, T.; Fujikawa, T.; Matsubayashi, N.; Fukumoto, T.; Yokoi, K.; Watanabe, I.; Ikeda, S. Anomalous Peak Intensities in the EXAFS of Polynuclear Molybdenum Compounds. Bull. Chem. Soc. Jpn. 1989, 62, 1791-1796. [CrossRef]

29. Furuta, S.; Miyanaga, T.; Watanabe, I. An abnormally large EXAFS Debye-Waller factor for a Mo-O bond in hexamolybdate. AIP Conf. Proc. 2007, 882, 141-143. [CrossRef]

30. Yan, L.; Lopez, X.; Carbo, J.J.; Sniatynsky, R.; Duncan, D.C.; Poblet, J.M. On the Origin of Alternating Bond Distortions and the Emergence of Chirality in Polyoxometalate Anions. J. Am. Chem. Soc. 2008, 130, 8223-8233. [CrossRef] [PubMed]

31. Miyanaga, T.; Sakane, H.; Watanabe, I. EXAFS Debye-Waller Factor and Ligand Exchange Reaction of Hydrated Metal Complexes. Bull. Chem. Soc. Jpn. 1995, 68, 819-824. [CrossRef]

32. Miyanaga, T.; Sakane, H.; Watanabe, I. Anharmonic Potential Derived from EXAFS of Hexaaqua Transition Metal Complexes. Phys. Chem. Chem. Phys. 2000, 2, 2361-2365. [CrossRef]

33. Akesson, R.; Pettersson, L.G.M.; Sandstrom, M.; Wahlgren, U. Theoretical Study on Water-Exchange Reactions of the Divalent and Trivalent Metal Ions of the First Transition Period. J. Am. Chem. Soc. 1994, 116, 8705-8713. [CrossRef] 